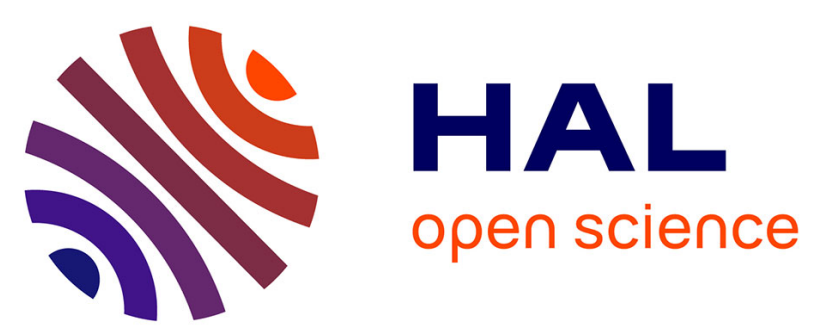

\title{
Fiber suspension in 2D nonhomogeneous flow: The effects of flow/fiber coupling for Newtonian and power-law suspending fluids
}

Dihya Mezi, Gilles Ausias, Suresh G Advani, Julien Férec

\section{To cite this version:}

Dihya Mezi, Gilles Ausias, Suresh G Advani, Julien Férec. Fiber suspension in 2D nonhomogeneous flow: The effects of flow/fiber coupling for Newtonian and power-law suspending fluids. Journal of Rheology, 2019, 63 (3), pp.405-418. 10.1122/1.5081016 . hal-02114272

\section{HAL Id: hal-02114272 \\ https://hal.science/hal-02114272}

Submitted on 29 Apr 2019

HAL is a multi-disciplinary open access archive for the deposit and dissemination of scientific research documents, whether they are published or not. The documents may come from teaching and research institutions in France or abroad, or from public or private research centers.
L'archive ouverte pluridisciplinaire HAL, est destinée au dépôt et à la diffusion de documents scientifiques de niveau recherche, publiés ou non, émanant des établissements d'enseignement et de recherche français ou étrangers, des laboratoires publics ou privés. 


\title{
Fiber suspension in 2D nonhomogeneous flow: The effects of flow/fiber coupling for Newtonian and power-law suspending fluids
}

\author{
Dihya Mezi, ${ }^{1}$ Gilles Ausias, ${ }^{1}$ Suresh G. Advani, ${ }^{2}$ and Julien Férec ${ }^{1, a)}$ \\ ${ }^{1}$ Univ. Bretagne Sud, UMR CNRS 6027, IRDL, F-56100 Lorient, France \\ ${ }^{2}$ Department of Mechanical Engineering, Center for Composite Materials, University of Delaware, \\ 101 Academy Street, Newark, Delaware 19716
}

(Received 13 November 2018; final revision received 11 March 2019; published 2 April 2019)

\begin{abstract}
A numerical study is presented for fiber suspension flows through a parallel plate channel and a planar 4:1 contraction. Besides examining a Newtonian suspending fluid, a non-Newtonian matrix exhibiting a pseudoplastic behavior and describing a power-law model is also investigated. Furthermore, instead of using orientation tensors for the macroscopic constitutive modeling, the proposed approach addresses the macroscopic scale by describing the fiber orientation state with the probability distribution function (PDF). It enables us to eliminate the error introduced due to the closure approximation when using orientation tensor description as our numerical scheme solves the PDF in both the spatial and configurational spaces. This allows us to correctly implement expressions for both the fiber extra stress, especially for the suspending matrix displaying a pseudoplastic behavior, and the fiber orientation state, describing the configuration. Hence, these two constitutive relations for suspensions are used to perform simulations in which flow and fiber orientation are fully coupled. Results are presented in planar geometries involving channel and 4:1 contraction flows. It is found that the coupling effect flattens the velocity profile for both suspending fluids but has a small impact on the fiber orientation distributions at the geometry outlets. However, in the corner region where a vortex is observed, its magnitude increases with the coupling and this enhancement is more pronounced for the Newtonian suspending fluid. The Newtonian viscosity model is replaced with the Carreau model and results are compared to a bi-viscosity model. It gives qualitatively correct results if no rapid fiber orientation change occurs along the streamlines. (C) 2019 The Society of Rheology.

https://doi.org/10.1122/1.5081016
\end{abstract}

\section{INTRODUCTION}

Rigid-fiber suspensions, such as short glass and carbon fibers in polymers, are encountered in several engineered products and represent an important class of non-Newtonian fluids. However, these systems exhibit a complex flow behavior due to the addition of fillers (i.e., fiber dynamics are affected by the fluid flow and conversely, the flow profile is modified by the presence of the particles) and the non-Newtonian character of the suspending matrix (i.e., a shear-thinning behavior is usually observed for composite materials made of polymers). Therefore, predicting and be able to control the orientation state of fiber suspensions are of major interest to design a successful manufacturing process and to predict the physical properties of the final product. This could be achieved by performing numerical simulations which uses a rigorous formulation to describe the coupled nature of flow and fiber orientation accurately.

Over the past four decades, various researchers have developed numerical strategies to perform fiber orientation predictions for flows inside complex geometries by addressing the coupling between the flow field and the fiber orientation distribution. This is a key element in predicting the behavior of fiber suspensions since the work of Evans [1],

\footnotetext{
a) Author to whom correspondence should be addressed; electronic mail: julien.ferec@univ-ubs.fr
}

who considered the flow of suspensions of rigid rodlike particles in a Newtonian fluid through simple geometries. Their results are based on the aligned-fiber approximation, which considers that fibers rotate rapidly to align with the streamlines. Lipscomb et al. [2] developed a numerical methodology to investigate the streamlines in a 4.5:1 axisymmetric contraction at low Reynolds number, using a Newtonian fluid with low concentrations of high aspect ratio fibers. Their results agreed quantitatively with their experimental observations despite the use of the aligned-fiber approximation. Ranganathan and Advani [3] considered the effect of fiberfiber interaction based on the Folgar-Tucker model [4] in an axisymmetric diverging radial flow. Kabanemi et al. [5] have highlighted the effect of both three-dimensional flow and fountain flow on fiber orientation during the injection molding of thick wall parts. Reddy and Mitchell [6] have developed a finite-element (FE) solver for fiber suspension flows in complex geometries, such as the benchmark 4:1 contraction problem, and were able to reproduce an enhancement in the magnitude of the zone of recirculation with increasing fiber concentration. Verweyst and Tucker [7] used a fully coupled solution to predict the flow of fiber suspensions through a variety of complex geometries including axisymmetric contractions, expansions, and center-gated disk. They also observed that the coupling enhances the corner vortex in the contraction, in quantitative agreement with experiments and calculations. In addition, they found that the aligned-fiber approximation is not quite correct in the 
recirculating region and in the axisymmetric expansion flow. Some experimental works confirmed that fibers modify the flow pattern of suspensions. Yasuda et al. [8] introduced short fibers in a Newtonian matrix and forced the suspension to flow through a channel with a rectangular cross section. The velocity profiles were measured with a magnetic resonance imaging velocimetry. They observed that fibers modify the velocity profile becoming flatter as the fiber volume fraction increases. As for the center-gated disk, Verweyst and Tucker [7] showed that the effects of coupling are modest and are only noticeable near the center of the disk. Mazahir et al. $[9,10]$ conducted coupled transient simulations to predict fiber orientation in a center-gated disk, employing slow orientation kinetics such as the reduced strain closure model [11]. They found that the coupling effect is very small when assessed against experimental data measured in the shell, transition, and core layers but an improvement is observed in the frontal flow region. A detailed review of the fundamentals and numerical simulations for predicting fiber orientation during injection molding process of polymer composites is given by Park and Park [12].

However, these approaches to fiber orientation modeling are based on using moment tensors of the fiber orientation probability distribution function (PDF) [13] and inevitability involve the well known problem of the choice of accurate closure approximations. Note that calculations using the aligned-fiber approximation do not require a closure approximation. Hence, some numerical strategies have been developed to circumvent this issue by solving directly the Fokker-Planck equation in both the spatial and configurational spaces. In rheology modeling of viscoelastic fluids (i.e., FENE model), the Fokker-Planck equation has been used to study the flow past a confined cylinder [14,15]. Moosaie and Manhart [16] proposed a two-way coupled direct simulation technique for the numerical problem of Brownian fiber suspension flows in complex geometries. This procedure yields a direct solution of the Fokker-Planck equation without requiring a closure model. Krochak et al. [17] investigated the effect of the two-way coupling between the flow field and the orientation state of rigid-fiber suspensions flowing through a tapered channel, where the orientation distribution function evolves according to a Fokker-Planck type equation. They showed that the orientation anisotropy is significantly different when the two-way coupling is included. A recent technique such as the proper generalized decomposition can also be used to treat problems involving spatial and configurational spaces $[18,19]$. These findings would suggest that the two-way coupling plays a fundamental role in predicting both the flow field and fiber orientation state during the flow of fiber suspensions. Furthermore, it is now conceivable to solve both the spatial and orientation domains when directly computing the orientation distribution function.

\section{THEORETICAL BACKGROUND OF FIBER SUSPENSIONS}

\section{A. Fiber orientation dynamics}

In practice, it is usual to describe the orientation of a single and rigid ellipsoid of revolution by the two angles $\theta$ and $\phi$ in a spherical coordinate system or equivalently by a unit vector $\mathbf{p}$ directed along its principal axis of symmetry [13]. As for its dynamics, Jeffery [20] solved the creeping flow equations for such a particle freely suspended in a Newtonian fluid assuming a linearly varying flow. It is found that the center of the particle translates with the local fluid velocity and its rotational motion is given by [including only the first two terms on the right-hand side of Eq. (1)]

$$
\dot{\mathbf{p}}=-\frac{1}{2} \boldsymbol{\omega} \cdot \mathbf{p}+\frac{\lambda}{2}(\dot{\gamma} \cdot \mathbf{p}-\dot{\gamma}: \mathbf{p p p})-\frac{C_{I}|\dot{\gamma}|}{\Psi} \frac{\partial \Psi}{\partial \mathbf{p}}
$$

In the above equation, $\boldsymbol{\omega}$ and $\dot{\gamma}$ are the vorticity and the deformation rate tensors, respectively (see [21] for their definitions). The shape factor is defined by $\lambda=\left(a_{r}^{2}-1\right) /$ $\left(a_{r}^{2}+1\right)$, where $a_{r}$ is the particle aspect ratio obtained from $a_{r}=L / D$, where $L$ and $D$ (or $2 R$ ) being its length and diameter, respectively. For a slender particle (i.e., cylindrical fiber), particle thickness can be ignored, setting $\lambda=1$ (this condition will be used through this work). The last term in the right-hand side of Eq. (1) was later introduced by Folgar and Tucker [4] to take into account particle interactions encountered in concentrated suspensions of non-Brownian fibers. $|\dot{\gamma}|$ denotes the magnitude of the effective deformation rate (i.e., the second invariant of the strain-rate tensor) and $C_{I}$ represents the interaction coefficient. Some authors [22,23] have attempted to relate $C_{I}$ with fiber aspect ratio and fiber volume fraction, $\phi_{f}$, and others [24-26] have considered it as an anisotropic rotary diffusion tensor. $\Psi$ is the PDF giving a complete and unambiguous description for representing the orientation state of a group of fibers.

For non-Newtonian suspending fluids, Laurencin et al. [27] investigated the fiber kinematics for non-Newtonian dilute fiber suspensions subjected to lubricated compression experiments using a microrheometer mounted in a synchrotron x-ray microtomograph. It allowed them to observe fast and in situ 3D imaging of the translation and rotation of fibers in the suspending fluid. They found that the Jeffery theory with its related affine assumption led to rather good estimations for the kinematics of a single fiber suspended in a non-Newtonian fluid (i.e., exhibiting a shear-thinning behavior) even by changing the macroscale compression strain rate. Thus, they concluded that the non-Newtonian character of the matrix has a minor effect on the kinematics of dilute fibers. Around the same time, based on the Giesekus formulation [28], Férec et al. [29] showed that Jeffery's equation (with the assumption of slender bodies) is satisfactory when considering that the suspending fluid follows a power-law behavior. This result was supported numerically in simple shear flow for a single ellipsoidal fiber with a large aspect ratio suspended in a power-law fluid [30]. Domurath et al. [31] also performed numerical simulations and showed that the particle motion for very large aspect ratio is not influenced by the non-Newtonian character of the suspending matrix given by a Carreau model.

From the PDF, it is useful to introduce its even-order moments (the odd-order ones being all zero) also known as 
orientation tensors [13]. For instance, $\langle\mathbf{p p}\rangle=\int_{\mathbf{p}} \mathbf{p p} \Psi d \mathbf{p}$ and $\langle\mathbf{p p p p}\rangle=\int_{\mathbf{p}} \mathbf{p p p p} \Psi d \mathbf{p}$ are the second- and fourth-order orientation tensors, respectively, and provide a more compact representation of the fiber orientation state in order to improve computational efficiency $\left(\int_{\mathbf{p}} \bullet \Psi d \mathbf{p}\right.$ refers to an average over all possible fiber orientations). The eigenvectors of $\langle\mathbf{p p}\rangle$ give the principal directions of fiber alignment, while the associated eigenvalues give the magnitude of fiber alignment in that direction. Following Eq. (1), Advani and Tucker [13] derived the evolution equation for $\langle\mathbf{p p}\rangle$ for particles suspended in a Newtonian fluid by taking into account fiber-fiber interactions

$$
\begin{aligned}
\frac{\partial}{\partial t}\langle\mathbf{p p}\rangle+\mathbf{u} \cdot \nabla\langle\mathbf{p p}\rangle= & -\frac{1}{2}(\boldsymbol{\omega} \cdot\langle\mathbf{p p}\rangle-\langle\mathbf{p p}\rangle \cdot \boldsymbol{\omega}) \\
& +\frac{\lambda}{2}(\dot{\gamma} \cdot\langle\mathbf{p p}\rangle+\langle\mathbf{p p}\rangle \cdot \dot{\gamma}-2 \dot{\gamma}:\langle\mathbf{p p p p}\rangle) \\
& -2 C_{I}|\dot{\gamma}|(\boldsymbol{\delta}-\alpha\langle\mathbf{p p}\rangle),
\end{aligned}
$$

where $\boldsymbol{\delta}$ is the identity tensor, $\mathbf{u}$ is the velocity vector of the suspending fluid, and $\nabla$ represents the differential operator with respect to the spatial coordinates. $\alpha$ equals 3 for threedimensional orientation and $\alpha$ equals 2 for planar orientation. It should be noted that this governing equation contains $\langle\mathbf{p p p p}\rangle$ and, therefore, a closure approximation to express $\langle\mathbf{p p p p}\rangle$ in terms of $\langle\mathbf{p p}\rangle$ is needed to solve the system of equations. Various closure approximations have been proposed and their accuracies are difficult to quantify, yielding to some questionable results [32-34]. As for a power-law fluid, Férec et al. [29] expressed the time evolution equation for a second-order conformation tensor without considering fiber interactions. Once again, a fourthorder tensor appears in the evolution equation for $\langle\mathbf{p p}\rangle$, the second-order tensor describing the rod microstructure. This time it involves a scalar potential leading to develop new closure approximations and exposing the results to criticism.

\section{B. Fiber stress expression}

Several approaches exist for deriving the total stress expression of fiber-filled composites. Among them, cell models (or self-consistent schemes) can give realistic constitutive equations with a simple but rigorous framework for slender bodies (i.e., particles with a high aspect ratio, $\lambda=1$ ). The seminal work was initiated by Batchelor $[35,36]$ for an elongational flow of a Newtonian fluid containing fibers parallel to the stretching direction, which was extended by Goddard [37,38] to include the strain-thinning behavior of the matrix by assuming a power-law model. Later, the cell model was updated to consider homogeneous flows for both the Newtonian and the power-law fluids. For instance, Souloumiac and Vincent [39] and later Férec et al. [29] established a stress expression for a fiber suspension, which includes the pseudoplastic character of the neat matrix modeled by a power-law relationship. Their results are in agreement with Gibson and Toll [40] and have the following form

$$
\begin{aligned}
\boldsymbol{\sigma}= & -P \boldsymbol{\delta}+K|\dot{\gamma}|^{m-1} \dot{\boldsymbol{\gamma}} \\
& +K|\dot{\gamma}|^{m-1} \phi_{f} \frac{a_{r}^{m+1}}{2^{m-1}(m+2)}\left[\frac{1-m}{m\left[1-(R / h)^{\frac{1-m}{m}}\right]}\right]^{m} \\
& \times \dot{\gamma}:\left\langle|\tilde{\dot{\gamma}}: \mathbf{p p}|^{m-1} \mathbf{p p p p}\right\rangle .
\end{aligned}
$$

In the above expression, the first term represents the total hydrostatic contribution, where $P$ is the isotropic fluid pressure. The second term is the suspending fluid contribution, where $K$ and $m$ are the consistency and the power-law index of the matrix, respectively. Finally, the last term on the righthand side refers to the particle contribution to the extra stress

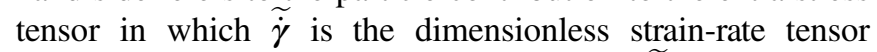
related to the strain-rate tensor by $\dot{\gamma}=|\dot{\gamma}| \widetilde{\dot{\gamma}}$ and $h$ corresponds to the average distance between fibers. In the limit as $m$ tends to unity, Eq. (3) yields the stress expression for a fiber suspension in a Newtonian fluid of viscosity $\eta_{0}$ by substituting $K$ with $\eta_{0}$

$$
\boldsymbol{\sigma}=-P \boldsymbol{\delta}+\eta_{0} \dot{\gamma}+\eta_{0} \phi_{f} \frac{a_{r}^{2}}{3 \log (h / R)} \dot{\gamma}:\langle\mathbf{p p p p}\rangle
$$

This rheological constitutive model for fiber suspensions was previously obtained by Dinh and Armstrong [41], and it is widely used in process simulations. When the fibers are randomly oriented, the concentration limits of interest in Eqs. (3) and (4) are given by $a_{r}^{-2}<\phi_{f}<a_{r}^{-1}$. This corresponds to the semiconcentrated regime and both limits decrease when increasing $a_{r}$. However, if the fibers are all parallel to one another, then the previous limits are replaced by $a_{r}^{-2}<\phi_{f}<1$.

As discussed above and when dealing with macroscopic descriptors, a closure approximation is required in Eq. (4) to express $\langle\mathbf{p p p p}\rangle$ in terms of $\langle\mathbf{p p}\rangle$ and some potentially tedious works are required to find a closed-form for Eq. (3). Therefore, a reasonable and theoretically exact approach consists of using a macroscopic descriptor such as the PDF. Up to now, it is well established that the main disadvantage of using the PDF to describe the orientation is that it makes the calculations to predict orientation in flowing suspensions very cumbersome [13]. But with the development of everfaster and higher-performing computers, such preconceived idea can be overcome leading to explore the efficiency of new rheological models without considering approximations such as closure relations. For fibers suspended in a fluid, the extra stress depends on both the deformation rate and the fiber orientation state. This leads to a two-way coupling between the solutions for velocity and orientation.

Understanding the evolution of the final fiber orientation state during the processing of the polymer fiber suspension into a polymer composite part is essential to predict and tailor the performance of the composite. Much of the focus have been dedicated to better predict the fiber dynamics in Newtonian fluids by considering the slow orientation kinetics 
and anisotropic rotary diffusion in concentrated systems, with some improvements but at the expense of introducing additional parameters. However, investigating the effect of the non-Newtonian character of the suspending fluid on the fiber orientation distribution has not been yet well explored and remains a formidable challenge. Hence, the main focus of this work is to handle this issue and, therefore, a general procedure is delineated to solve the flow and fiber orientation in a coupled way. Furthermore, the developed tool is capable of analyzing and testing more realistic physics into the modeling. This paper is organized as follows. In Sec. III, the theoretical fundamentals of the numerical model is outlined. Then, in Sec. IV, computational results in 2D are presented and concern the fiber suspension flows in the Newtonian and the non-Newtonian matrices through a parallel plate channel and a planar 4:1 contraction, respectively. Section $\mathrm{V}$ aims to investigate the use of the Carreau law by considering a bi-viscosity model, and finally, Sec. VI concludes the paper.

\section{MODEL DEFINITION}

\section{A. Continuity equation for the PDF}

Let $\Psi$ be the PDF for a fiber in the configuration $(\mathbf{x}, \mathbf{p}, t)$, where $\mathbf{x}$ designates the position vector for its center of gravity, $\mathbf{p}$ its orientation, and $t$ the time. Therefore, the location and orientation of a single and rigid fiber at a time $t$ is given by specifying the Cartesian components $x, y, z$ for $\mathbf{x}$ and $p_{x}, p_{y}, p_{z}$ for $\mathbf{p}$, respectively. This set of six coordinates can be represented as a particular system point in a sixdimensional spatial and configurational space. Thus, for a medium containing a large number of particles, the time rate of change of system points with a six-dimensional hypercube of volume $V=\Delta x \Delta y \Delta z \Delta p_{x} \Delta p_{y} \Delta p_{z}$ is $\dot{\Psi} \Delta x \Delta y \Delta z \Delta p_{x} \Delta p_{y} \Delta p_{z}$. The rate at which system points enter the hypercube is $\left.(\dot{x} \Psi)\right|_{x} \Delta y \Delta z \Delta p_{x} \Delta p_{y} \Delta p_{z}-\left.(\dot{x} \Psi)\right|_{x+\Delta x} \Delta y \Delta z \Delta p_{x} \Delta p_{y} \Delta p_{z} \quad$ plus 10 additional terms describing the position and orientation change in the other five coordinate directions. These two expressions can now be equated and then divided by $V$. When $\Delta x, \Delta y$, etc., tend to zero, it results in

$$
\dot{\Psi}=-\nabla_{\mathbf{x}} \cdot(\dot{\mathbf{x}} \Psi)-\nabla_{\mathbf{p}} \cdot(\dot{\mathbf{p}} \Psi)
$$

which is called the equation of continuity for $\Psi . \dot{\Psi}$ represents the partial time derivative of the PDF and more generally, the over-dot symbol refers to the partial time derivative. The symbol $\nabla_{\mathbf{x}}$ is the useful differential operator in position space, and $\nabla_{\mathbf{p}}$ corresponds to the differential operator on the surface of a unit sphere, such as $\nabla_{\mathbf{p}}(\bullet)=\mathbf{e}_{\theta} \frac{\partial \bullet}{\partial \theta}+\mathbf{e}_{\phi} \frac{\partial \bullet}{\sin \theta \partial \phi}$, with $\mathbf{e}_{\theta}$ and $\mathbf{e}_{\phi}$ being the polar and azimuthal unit vectors, respectively. Note that the first right-hand side term of Eq. (5) becomes null in the case of steady homogenous flows.

\section{B. Configurational discretization by using a finite volume method}

When the time evolution for $\mathbf{p}$, given by Eq. (1), is inserted into the continuity equation, Eq. (5), one obtains the so-called Smoluchowski equation also known as the FokkerPlanck equation (the latter designation will be used in the following with the acronym FP). Similar to the rotational diffusion encountered in Eq. (1) (i.e., the term including $C_{I}$ ), some translational diffusion (parallel and perpendicular to the particle axis) may occur for the time evolution of the center of mass of the fiber. As a homogeneous system is considered, it will remain homogeneous even if the orientational distribution is not isotropic [42]. Thus, the translational diffusion terms can be discarded and the center of mass of the fiber is moving with the fluid velocity (affine motion). In this form, no analytical solution is available for the FP equation, especially when both the spatial configuration is taken into account and fiber interactions are considered $\left(C_{I} \neq 0\right)$. The difficulty is that the FP equation must be solved in both physical and configurational spaces, and this requires a suitable discretization procedure for all relevant variables, namely, space $\mathbf{x}$, configuration $\mathbf{p}$, and time $t$. Thus, in the following, only problems dealing with steady-state regime are investigated, but even under this assumption, a numerical approach is still required to solve the equation below

$$
\mathbf{u} \cdot \nabla_{\mathbf{x}} \Psi+\nabla_{\mathbf{p}} \cdot(\dot{\mathbf{p}} \Psi)=0
$$

To obtain Eq. (6), the notation for the velocity vector $\dot{\mathbf{x}}$ has been replaced by $\mathbf{u}$ and the requirement for incompressible flow has been used. Furthermore, this work focuses on a 2D problem reducing the problem to three variables issue, i.e., two spatial Cartesian coordinates ( $x$ and $y$ ) and one configurational coordinate $(\phi)$ related to the fiber orientation distribution. This multidimensional problem imposes a mesh for the fiber orientation angle $\phi$ domain at each spatial node. Hence, a finite volume method (FVM) is used to discretize the partial differential equation, Eq. (6), in the configurational space. The FVM is recognized for its local conservativeness property needed to fulfill the normalization condition for the PDF, which is included in the formalism of the model. Following the work of Férec et al. [43], the numerical approach has been updated to deal with a two-dimensional representation of fiber orientation state (i.e., planar orientation state), leading to considering the angle for particle orientation, $\phi$. In this context, all the possible orientations describe half circle of unit radius since a fiber head is undistinguished from its tail, and it results in the following periodic boundary conditions: $\Psi(\phi)=\Psi(\phi+\pi)$. Thus, the perimeter of the half circle has been discretized into $N$ equal intervals of length $\Delta \phi=\pi / N$. In the configurational space, an upwinding powerlaw scheme is used to solve the convection-diffusion problem [44]. Therefore, the discretized form for nodal points $P, E$, and $W$ (see Fig. 1) of the FP equation, Eq. (6), is

$$
\Delta \phi \mathbf{u} \cdot \nabla_{\mathbf{x}} \Psi_{P}+a_{P} \Psi_{P}-a_{W} \Psi_{W}-a_{E} \Psi_{E}=0
$$

The Appendix presents the expressions for the coefficients $a_{E}, a_{W}$, and $a_{P}$.

At each node of the mesh of the volume, $N$ components of the PDF are known or computed. For $N$ directions called $\mathbf{p}_{i}$, the value of the probability to find fiber in this direction, $\Psi_{i}$, is also known or computed. Terms such as 


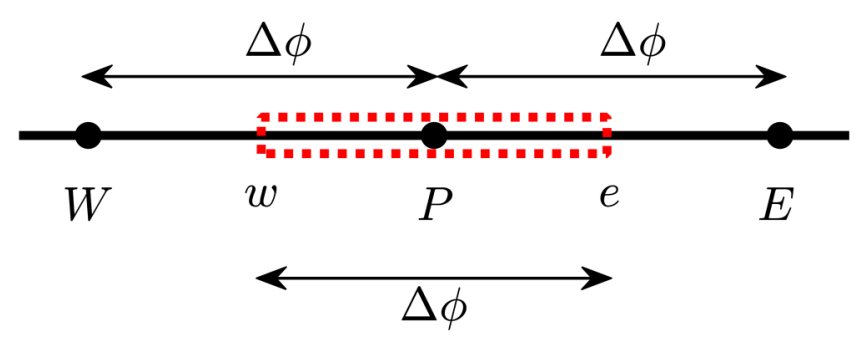

FIG. 1. Control volume used to perform flux balances for the PDF (represented by the red-dotted box). The edges of the control volume labeled as $e$ and $w$ refers to east and west directions.

$\langle\mathbf{p p p p}\rangle$ are computed with the use of the $N$ components of PDF substituting the integral for a sum as

$$
\langle\mathbf{p p p p}\rangle=\int_{\mathbf{p}} \mathbf{p p p p} \Psi d \mathbf{p} \approx 2 \Delta \phi \sum_{i=1}^{N} \mathbf{p}_{i} \mathbf{p}_{i} \mathbf{p}_{i} \mathbf{p}_{i} \Psi_{i}
$$

In the same manner, the term into brackets in Eq. (3) can be computed as

$$
\left\langle\widetilde{\dot{\gamma}}:\left.\mathbf{p p}\right|^{m-1} \mathbf{p p p p}\right\rangle \approx 2 \Delta \phi \sum_{i=1}^{N}\left|\widetilde{\dot{\gamma}}: \mathbf{p}_{i} \mathbf{p}_{i}\right|^{m-1} \mathbf{p}_{i} \mathbf{p}_{i} \mathbf{p}_{i} \mathbf{p}_{i} \Psi_{i}
$$

In this work, values of conformation tensors are only the result of post treatment.

\section{Numerical implementation}

COMSOL Multiphysics 5.2 is used to solve the full problem (i.e., flow field and fiber orientation) since its framework allows coupling the existing laminar flow interface with partial differential equation (PDE) interfaces, required for implementing the extra fiber stress. As the discretization in the configurational space involves $N$ nodal points, the coupled system of PDEs is represented by using the matrix forms of coefficients. The unknowns are the values for the PDF at each node in the configurational space and, therefore, it involves $N$ coefficient forms. Equation (7) is a convection equation in the spatial domain and is then stabilized by adding a numerical isotropic diffusion term. In this case, the diffusion coefficient is an artificial diagonal $N \times N$ matrix with $\Delta \phi c_{\text {num }}$ on its diagonal. This provides the only spatial diffusion for the FP equation. Under this assumption, Eq. (7) becomes

$\Delta \phi \mathbf{u} \cdot \nabla_{\mathbf{x}} \Psi_{P}+\Delta \phi c_{\text {num }} \Delta_{\mathbf{x}} \Psi_{P}+a_{P} \Psi_{P}-a_{W} \Psi_{W}-a_{E} \Psi_{E}=0$,

where $\Delta_{\mathbf{x}}$ represents the Laplacian operator. This equation is implemented in Comsol Multiphysics by taking advantage of the similarity with the coefficient form PDE, presented in the software. Hence, coefficients in the above equation are identified with the ones for the convection-diffusion problem. Note that the absorption coefficients are introduced with respect to the connectivity table from the mesh in the configurational domain.

\section{Model statement}

The two constitutive equations for a Newtonian or a power-law suspending fluids must be solved together with the continuity equation for an isothermal and incompressible fluid, and the Cauchy momentum equation in the limit of creeping flow with negligible body forces

$$
\begin{aligned}
& \nabla_{\mathbf{x}} \cdot \mathbf{u}=0, \\
& \nabla_{\mathbf{x}} \cdot \boldsymbol{\sigma}=\mathbf{0},
\end{aligned}
$$

where $\mathbf{u}$ is the velocity vector and $\boldsymbol{\sigma}$ denotes the total stress tensor, given by relations (3) or (4). The FP equation, Eq. (10), provides the rod orientation dynamics equation represented by $\Psi$, from which the tensorial terms in angular brackets, arising in Eqs. (3) and (4), are straightforwardly numerically computed. It is recalled that no closure approximation is required thanks to this framework.

All the computations were carried out on a laptop HP EliteBook Folio 1040 G3 Notebook PC Series with Intel(R) Core(TM) i7-6600U CPU (2.6GHz) and 16GB RAM and did not exceed $3 \mathrm{~h}$ of CPU time. The Comsol Multiphysics default mesh settings, i.e., type physics-controlled mesh sizes, were used. The mesh is optimized in order to generate boundary layers around no-slip boundaries and edges. Boundary layers (thin quadrangles) are useful along all boundaries across where strong gradients of the velocity develop. Hence, considering both accuracy and computational time, a fine mesh size was selected for all the computations done in this paper.

Also, in all computations presented in this work, the parameters have the following values: $a_{r}=20, \phi_{f}=10 \%$, $h / R=\sqrt{\pi / \phi_{f}}$ (this condition corresponds to the case of aligned fibers and is kept constant over the different simulations even if the particles orient), $\eta_{0}=1 \mathrm{Pas}, K=1 \mathrm{Pas}^{m}$, and $m=0.3$. An interaction coefficient of $C_{I}=0.01$ was selected for the example problem and the artificial numerical diffusion coefficient, $c_{\text {num }}$, is set to $10^{-5} \mathrm{~m}^{2} / \mathrm{s}$ for the spatial diffusion in the FP equation, Eq. (10). The value of the parameters are summarized in Table I.

The proposed models do not account for any particle migration that is known to occur near boundaries because of the fiber-wall contacts. This first study aims to investigate the bulk flow of fiber suspensions in the Newtonian and the power-law suspending fluids; however, considering the depletion of particles near the boundary could be the focus for a future work.

TABLE I. Parameter values used for the numerical simulations.

\begin{tabular}{lccc}
\hline \hline Model parameters & Symbol & Value & Unit \\
\hline Fiber volume fraction & $\phi_{f}$ & 0.1 & - \\
Fiber aspect ratio & $a_{r}$ & 20 & - \\
Suspending fluid viscosity & $\eta_{0}$ & 1 & $\mathrm{~Pa} \mathrm{~s}$ \\
Suspending fluid consistency & $K$ & 1 & $\mathrm{~Pa} \mathrm{~s}^{m}$ \\
Power-law index & $m$ & 0.3 & - \\
Interaction coefficient & $C_{I}$ & 0.01 & - \\
\hline
\end{tabular}




\section{COMPUTATIONS ON 2D DOMAINS}

Two 2D computational domains are considered: a channel domain, where the flow gradient is predominantly shearlike, and a 4:1 contraction domain, displaying regions with shear flow, contraction/elongation flow, and recirculating flow in the corner. The advantage of these configurations is that the flow is two-dimensional and the orientation state is described by just the angle $\phi$. This allows us to investigate the effect of orientation on the kinematics of a nonhomogeneous flow. This will lead us to better understand the nature of the coupling between the flow and fiber orientation, for both the Newtonian and the power-law suspending matrices. For both geometries, the boundary conditions involve the following cases: At the entrance, a laminar inflow (BC1) is assumed with imposing a Dirichlet condition for the velocity such as its $x$-component is given by $u_{x}^{N}=\frac{3}{2} \bar{U}\left[1-(y / H)^{2}\right]$ for the Newtonian suspending fluid and $u_{x}^{P L}=\frac{2 m+1}{m+1} \bar{U}\left[1-(|y| / H)^{(m+1) / m}\right]$ for the power-law ones, respectively (the average velocity, $\bar{U}$, is set to $1 \mathrm{~m} / \mathrm{s}$ ). The outlet boundary is a pressure outlet condition, which includes a normal flow and a suppressed backflow (BC2). A zero-slip boundary condition (BC3) is applied to the walls and a symmetry boundary condition (BC4) which states that there is no flow across the boundary and zero tangential stress along the line of symmetry. Finally at the channel entrance, fibers are assumed to present a random-planar distribution leading to impose a Dirichlet-type boundary condition, $\Psi=1 /(2 \pi)$, for the FP equation (this equation is hyperbolic and, therefore, only requires a boundary condition at the inlet).

\section{A. Planar channel flow}

As the first example, the flow of a fiber suspension is assumed to occur in a planar channel geometry, corresponding to a laminar flow between two parallel plates. The computation domain is a rectangle with a length of $3 H$ in the $x$-direction and a height of $2 H$ in the $y$-direction, where $H=1 \mathrm{~m}$. Due to the symmetry about the $y=0$ axis, only the half rectangular duct is considered (see Fig. 2). The boundary at $x=0$ and at the opposite boundary at $x=3 H$ are a laminar inflow and an pressure outlet conditions, respectively. The top wall at $y / H=1$ has a zero-slip boundary condition and there is a symmetry boundary condition at $y / H=0$. This flow is not a true filling calculation but is intended to highlight the differences between the two

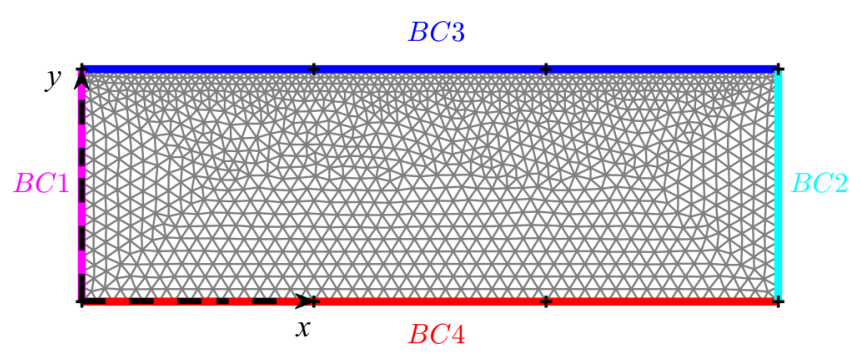

FIG. 2. FE mesh for planar channel: $\mathrm{BC} 1$, laminar inflow; $\mathrm{BC} 2$, pressure outlet; $\mathrm{BC} 3$, zero - slip condition; and $\mathrm{BC} 4$, symmetry condition. constitutive models when the flow field and fiber orientation are coupled or not. The domain is discretized using 2451 elements as shown in Fig. 2.

First, the Newtonian suspending fluid with no coupling between the flow field and the fiber orientation is examined. The flow field is assumed to be steady and fully developed. In this case, the streamlines are parallel to the walls, hence, this is a pure shear flow except on the bottom boundary, where the velocity gradient vanishes. Therefore, since the velocity gradient is constant along a streamline, each infinitesimal fluid element experiences a constant shear flow. It should be recalled that fiber orientation predictions made under these assumptions do not correspond to any physical process. However, the results provide information on the effect of flow on fiber orientation. For that purpose, the usefulness of these calculations is to check the implementation of the computer code.

The background color in Fig. 3 symbolizes the generalized shear rate distribution in the fluid domain (which equals to shear rate for this flow) and the white unfilled ellipses describe the average fiber orientation. The eigenvalues and the eigenvectors of $\langle\mathbf{p p}\rangle$ give the two major axes of the ellipse and indicate the degree of orientation along these directions. For instance, the ellipse becomes a circle for a planar random orientation and elongates into a line along the major axis for fiber orientation with a preferential alignment in that particular direction. Thus, fibers along the fixed wall become highly oriented and obtain the steady state instantly and fibers further away from the bottom boundary must travel quite a bit downstream to attain the steady state. As predicted by the Folgar-Tucker model, the nonzero value for $C_{I}$ tends to randomize the orientations leading to not align all the fibers along the streamlines.

The same steady state can also be obtained by performing steady single-point calculations (SPCs). Indeed, an observer moving with the fluid allows one to calculate the dynamic orientation history along a streamline. Following Férec et al. [29], the $\left\langle p_{x} p_{x}\right\rangle$ and $\left\langle p_{x} p_{y}\right\rangle$ components are directly computed from the FP equation for planar orientations with the assumption of homogeneous shear flow and with prescribing a constant velocity gradient value along a streamline. To perform this calculation, the half circle of unit radius is discretized into 100 equal intervals of length, whereas the FE scheme considers only $N=30$ elements for the

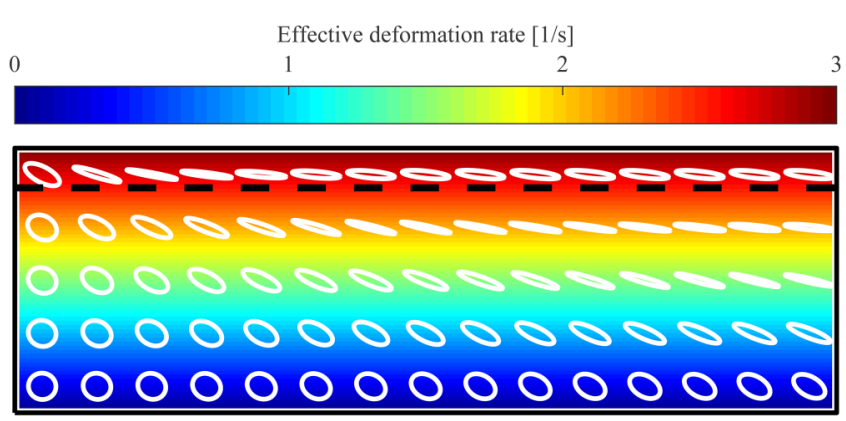

FIG. 3. Generalized shear rate distribution of fluid domain in the $x y$-plane for a Newtonian fluid (uncoupled solution). Ellipsoids represent the average fiber orientation and eigenvalues are scaled with $1 / 10$ for better visualization. 


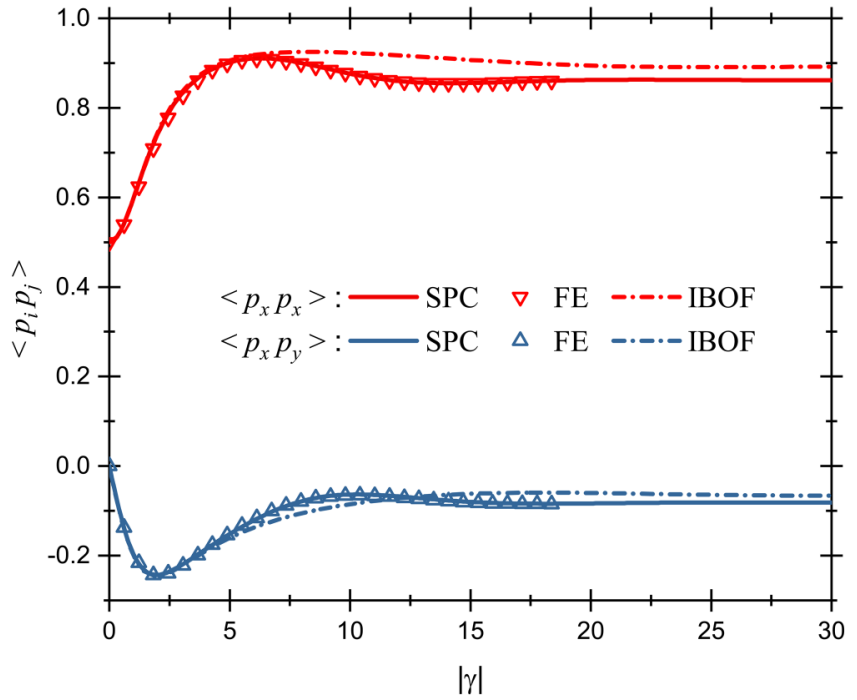

FIG. 4. Comparison of the FE to single-point calculations (SPC) of $\left\langle p_{x} p_{x}\right\rangle$ and $\left\langle p_{x} p_{y}\right\rangle$ in parallel plate channel for the streamlines along the line $y / H=0.85$. Predictions obtained with the IBOF closure approximation [45] are also shown.

configurational space. Therefore, to directly compare the FE results with SPCs, orientation state components are expressed as a function of total strain, $|\gamma|$. Recall that $\left\langle p_{x} p_{x}\right\rangle$ is proportional to the magnitude of the alignment and that $\left\langle p_{x} p_{y}\right\rangle$ indicates the direction of alignment. Figure 4 shows a comparison of FE and transient SPCs for a streamline, here represented in Fig. 3 by the black dashed line parallel to the symmetry centerline at $y=0.85 H$. The FE results are found to be consistent with the SPCs in providing the right dynamics and an accurate steady state and, hence, validates the implementation of the FE code and also the choice of $N=$ 30 elements as sufficient for the configurational mesh.

A major concern is what would be the fiber orientation distribution if Eq. (2) is used. This requires a closure approximation in order to express $\langle\mathbf{p p p p}\rangle$ as a function of $\langle\mathbf{p p}\rangle$. As $\langle\mathbf{p p p p}\rangle$ cannot be uniquely defined in terms of $\langle\mathbf{p p}\rangle$, the eigenvalue based orthotropic fitted closure based on the works of Cintra and Tucker [32] or the invariant based orthotropic fitted closure (IBOF) of Chung and Kwon [45] are typically used to approximate the coefficients used based on typical flow fields. The IBOF closure is known to be relevant and is therefore used for the calculations. Hence, Fig. 4 shows that the $\left\langle p_{x} p_{x}\right\rangle$ component given by the IBOF closure exhibits a larger overshoot and an overestimate of the steady plateau value (relative error of $3.5 \%$ ). As for the component $\left\langle p_{x} p_{y}\right\rangle$, the prediction is less accurate as the dynamic response is longer as compared to the PDF solution and the steady plateau value is underestimated (relative error of $18.6 \%$ ).

Then, the power-law suspending matrix is explored, still considering the decoupled solution between the fluid flow and the orientation evolution (see Fig. 5). In a planar channel, the power-law character of the fluid affects the velocity profile and a low value of the power-law index flattens the velocity profile (as $m$ tends to zero, the velocity profile becomes a pluglike). In this flattened region, the shear

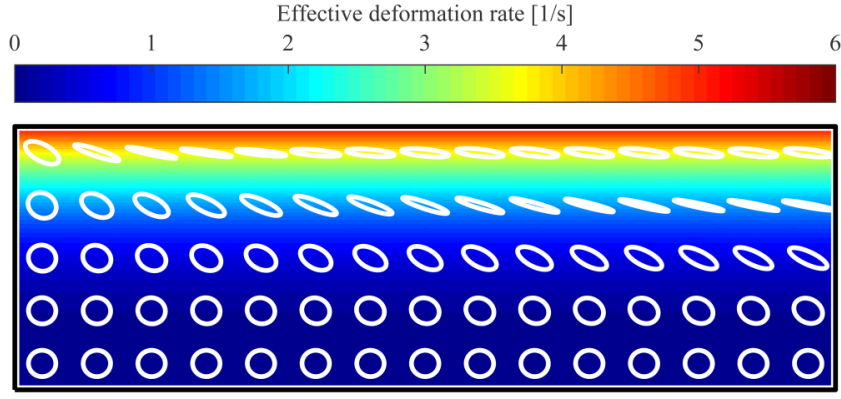

FIG. 5. Generalized shear rate distribution of fluid domain in the $x y$-plane for a power-law fluid with a power-law index of $m=0.3$ (uncoupled solution). Ellipsoids represent the average fiber orientation and eigenvalues are scaled with $1 / 10$ for better visualization.

rate is nearly zero and fibers are advected through the flow without changing their orientation distribution (the ellipses maintain their circular shapes). Also, close to the wall fibers become highly oriented and obtain the steady-state quasi-instantly, similar to the Newtonian case.

When the coupling effect is considered, the suspension becomes a non-Newtonian fluid since the fiber stress contribution is taken into account in the momentum equation. Indeed, viscosity becomes anisotropic when the average orientation of the fibers leaves their random-planar configuration. Hence, due to the non-Newtonian nature of the flow, the velocity profile is expected to change through the crosssections of the channel. Figure 6 depicts the numerical results when assuming a Newtonian suspending fluid and a coupling between the flow field and fiber orientation. Starting from a prescribed parabolic velocity profile at the inlet, the velocity profile tends to flatten out along cross-sections through the duct. Hence, due to the flat velocity profile, the effective deformation (i.e., shear rate) decreases in the central region of the channel when compared with the uncoupled solution. Furthermore, near the inlet, the streamline divergence leading to extension in the $y$-direction and thus more aligned fibers in the perpendicular direction to the flow.

The PDF can be recovered once the fiber orientation tensors are known thanks to a Fourier series expansion, where the coefficients transform as tensors under rigid rotations of the material element [46]. This property is then exploited to evaluate how the truncation limits given by orientation tensors can effectively describe the fiber orientation

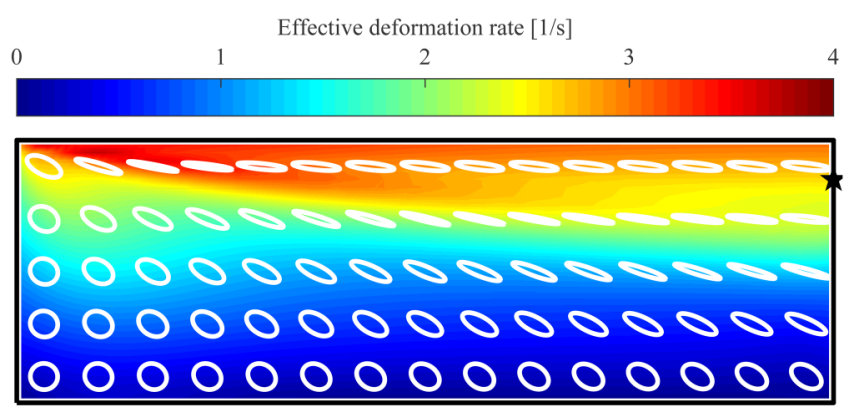

FIG. 6. Generalized shear rate distribution of fluid domain in the $x y$-plane for a Newtonian fluid (coupled solution). Ellipsoids represent the average fiber orientation and eigenvalues are scaled with 1/10 for better visualization. 
distribution. For planar orientation states, a fourth-order truncation $\bar{\Psi}_{4}$ is given by [13]

$$
\bar{\Psi}_{4}=\frac{1}{2 \pi}+\frac{2}{\pi} f_{2}: V_{2}+\frac{8}{\pi} f_{4}:: V_{4},
$$

where :: is the scalar product (quadruple contraction) of two fourth-order tensors. In the above relationship, $\boldsymbol{V}_{\mathbf{2}}$ and $\boldsymbol{V}_{4}$ represent the second- and the fourth-order deviatoric fiber orientation tensors defined in terms of index notation as

$$
\begin{gathered}
V_{i j}=\left\langle p_{i} p_{j}\right\rangle-\frac{1}{2} \delta_{i j}, \\
V_{i j k l}=\left\langle p_{i} p_{j} p_{k} p_{l}\right\rangle-\frac{1}{6}\left(\delta_{i j}\left\langle p_{k} p_{l}\right\rangle+\delta_{i k}\left\langle p_{j} p_{l}\right\rangle \delta_{i l}\left\langle p_{j} p_{k}\right\rangle+\delta_{j k}\left\langle p_{i} p_{l}\right\rangle+\delta_{j l}\left\langle p_{i} p_{k}\right\rangle+\delta_{k l}\left\langle p_{i} p_{j}\right\rangle\right)+\frac{1}{24}\left(\delta_{i j} \delta_{k l}+\delta_{i k} \delta_{j l}+\delta_{i l} \delta_{j k}\right),
\end{gathered}
$$

and $f_{2}$ and $f_{4}$ are their associated tensor basis functions of $\mathbf{p}$ such as

$$
\begin{gathered}
f_{i j}=p_{i} p_{j}-\frac{1}{2} \delta_{i j} \\
f_{i j k l}=p_{i} p_{j} p_{k} p_{l}-\frac{1}{6}\left(\delta_{i j} p_{k} p_{l}+\delta_{i k} p_{j} p_{l}+\delta_{i l} p_{j} p_{k}+\delta_{j k} p_{i} p_{l}+\delta_{j l} p_{i} p_{k}+\delta_{k l} p_{i} p_{j}\right)+\frac{1}{24}\left(\delta_{i j} \delta_{k l}+\delta_{i k} \delta_{j l}+\delta_{i l} \delta_{j k}\right) .
\end{gathered}
$$

Note that the second-order truncation $\widehat{\Psi}_{2}$ is obtained by considering the first two terms of Eq. (13). At the channel entrance, the random-planar distribution sets $\Psi=1 /(2 \pi)$ leading to express this fiber orientation state by a black dashed line in Fig. 7. This fiber conformation is perfectly described by any order of truncation. The three other curves in Fig. 7 show some fiber orientation distributions at the channel outlet at $y=0.85 H$ (represented by a black pentagram in Fig. 6). The red curve (square-shaped dots) in Fig. 7 represents the fiber distribution obtained from our numerical simulation. With regard to Fig. 6 with ellipsoidal

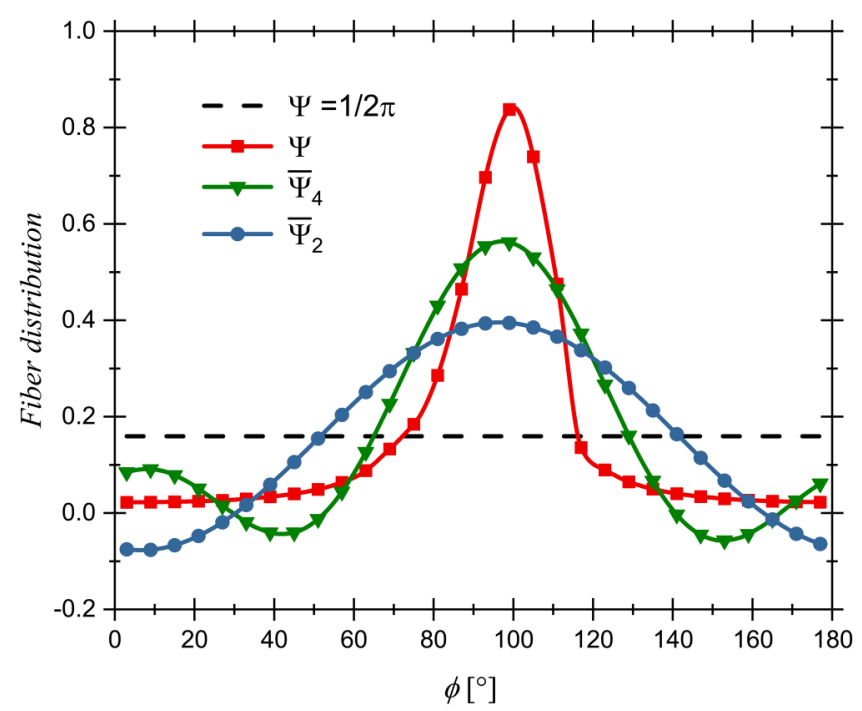

FIG. 7. PDF compared to recovered distribution functions using secondorder and fourth-order tensors at $x=3 H$ and $y=0.85 H$ (represented by a pentagram in Fig. 6) for a Newtonian fluid (coupled solution). representation, most fibers are aligned in the flow direction (the flow direction being at an angle of $\phi=90^{\circ}$ ) and, consequently, few of them are oriented along the flow-gradient direction (corresponding to $\phi=0^{\circ}$ and its opposite being at $\phi=180^{\circ}$ ). The green curve (triangle-shaped dots) in Fig. 7 is the fourth-order truncation $\bar{\Psi}_{4}$, where the deviatoric fiber orientation tensors are computed from $\Psi$. It leads that information about the fourth-order, given by $\left\langle p_{i} p_{j} p_{k} p_{l}\right\rangle$, is insufficient to represent quasialigned distributions, which represents most steady-state solutions for flow-induced orientation. In other words, handling orientation tensors up the fourth-order, which commonly done in flow processing, provides some erroneous values in describing fiber orientation states. It is obvious that inaccurate representations are observed when using the second-order truncation $\bar{\Psi}_{2}$ (see the blue curve with circle-shaped dots in Fig. 7).

The numerical results for a power-law suspending fluid by considering a coupled flow kinematics and fiber evolutions are presented in Fig. 8. Once again, the coupling effect tends to more flatten the velocity profile when a laminar flow, identical to the uncoupled case, is imposed at the entrance. Thus, in the central region near the centerline where the shear rate is mostly null, more fibers are convected through the flow when compared to the uncoupled solution.

To illustrate the flatness of the velocity profile with the coupling effect and the nature of the suspending fluid, Fig. 9 shows the velocity profiles at the outlet (i.e., $x=3 H$ ). Note that both red curves also correspond to the velocity profiles at the inlet (i.e., $x=0$ ) on the left side for the Newtonian suspending fluid and on the right side for the power-law suspending fluid, respectively. As already stated, the coupling tends to flatten the velocity profiles and this effect is noticeable for both suspending fluids. This flattening behavior is 


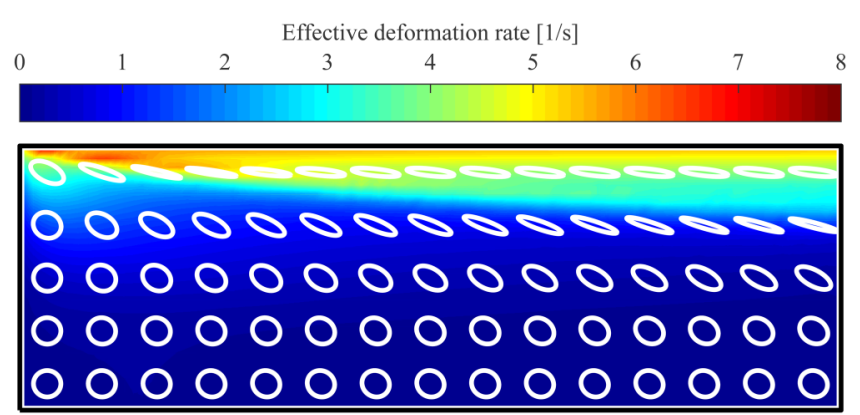

FIG. 8. Generalized shear rate distribution of fluid domain in the $x y$-plane for a power-law fluid with a power-law index of $m=0.3$ (coupled solution). Ellipsoids represent the average fiber orientation and eigenvalues are scaled with $1 / 10$ for better visualization.

induced by the fiber orientation distribution in the sense as higher is the fiber alignment lower is the shear viscosity of the suspension. Hence close to the wall where fibers are strongly aligned, the viscosity is lower than at the centerline leading to flatten the velocity profile.

The previous results suggest that taking into account the coupling effect has a minor impact on the fiber orientation distribution for the fluid flow in a planar channel. However, the pressure drop increases considerably when considering the coupling. Figure 10 depicts the computed pressure drop along the symmetrical axis (i.e., $y / H=0$ ) for the four investigated systems, in which the largest enhancement of the pressure drop is obtained for the Newtonian suspending fluid. Also shown in Fig. 10 are the analytical solutions given by the Hagen-Poiseuille equation for both unfilled suspending fluids.

\section{B. Planar contraction flow}

The second example consists in investigating the flow for a fiber suspension in a 4:1 contraction. A lot of numerical studies have focused on suspension flows through

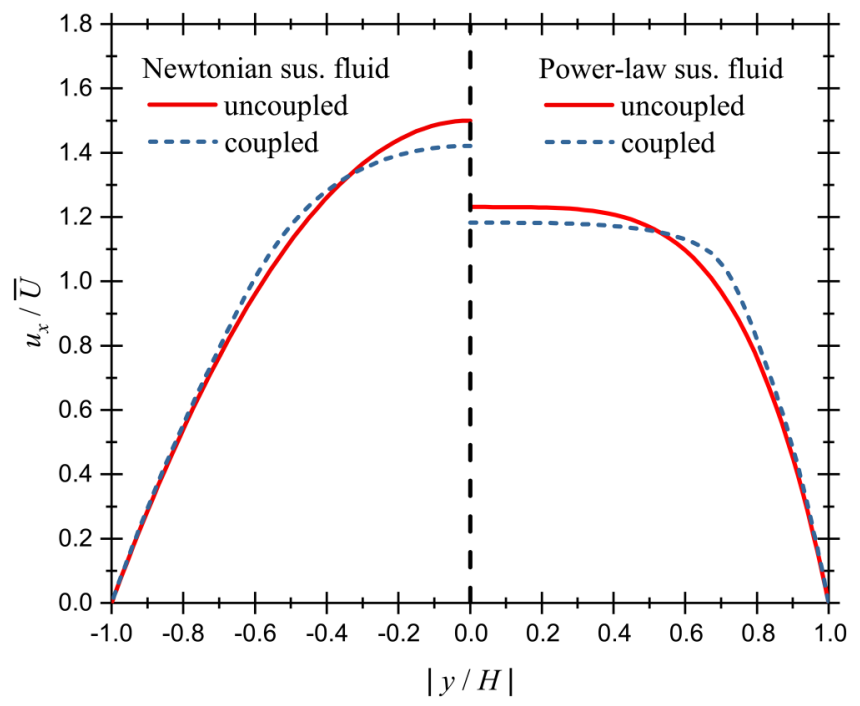

FIG. 9. Velocity profiles along the $y$-direction at the channel exit. On the left, the Newtonian suspending fluid. On the right, the power-law suspending fluid.

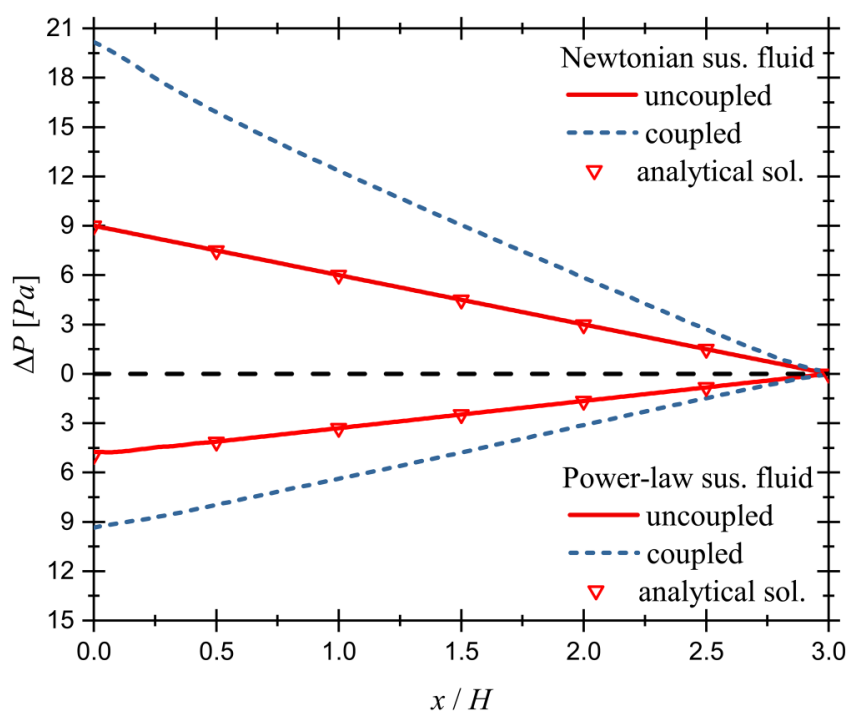

FIG. 10. Computed pressure drop along the symmetrical axis in the planar channel.

channel with abrupt expansion [47] or contraction [2]. Such flows are often encountered in a real molding process and therefore represent some relatively important benchmark cases. In fact, the contraction flow and its reverse (i.e., expansion flow) are geometries where the coupling has a large effect on the flow pattern [48]. In this case, the computational domain consists of two connected rectangles and is discretized using 2161 elements, as shown in Fig. 11. The boundary at $x=-6 \mathrm{~m}$ is the inlet and the outlet is defined at the opposite end, that is, at $x=6 \mathrm{~m} . y / H=0$ corresponds to the centerline of symmetry and the remaining boundaries are treated as walls.

The numerical results for an uncoupled solution when considering a Newtonian suspending fluid is depicted in Fig. 12. This time, the background color represent the magnitude of the $x$-component of the velocity and some streamlines are also visualized for a better understanding. A vortex is observed near the corner at $x=0$ and its effect is clearly seen in the fiber orientation field, where most fibers are parallel to the streamlines. This is highlighted in Fig. 16(a), in which a magnification of the black dashed-line rectangle zone is done. The alignment is not perfect as the fiber-fiber interaction coefficient is not zero (i.e., $C_{I} \neq 0$ ). Furthermore, fibers close to the centerline are subjected to elongational flow, especially near the entry region, and consequently align them in the flow direction.

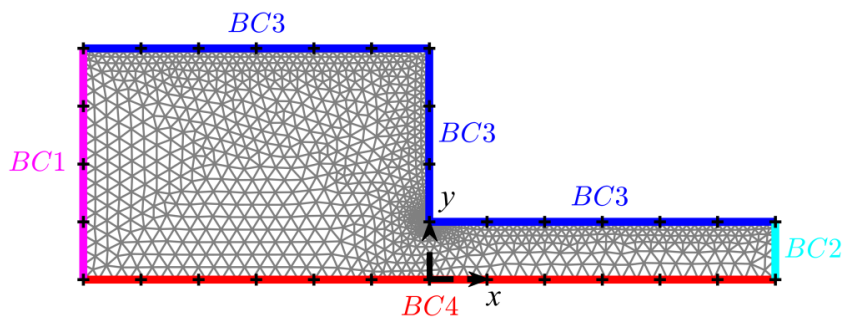

FIG. 11. $\mathrm{FE}$ mesh for $4: 1$ contraction: $\mathrm{BC} 1$, laminarinflow; $\mathrm{BC} 2$, pressureoutlet; $\mathrm{BC} 3$, zero - slipcondition; and $\mathrm{BC} 4$, symmetrycondition. 


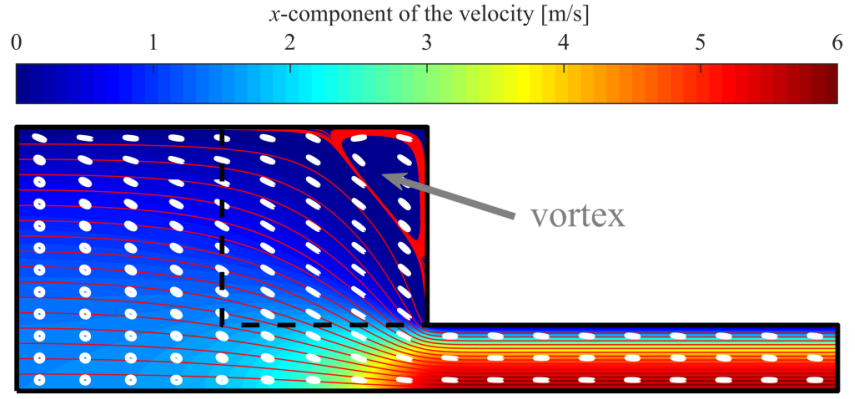

FIG. 12. $x$-component velocity distribution of fluid domain in the $x y$-plane for a Newtonian fluid (uncoupled solution). Ellipsoids represent the average fiber orientation, and eigenvalues are scaled with 1/10 for better visualization. Red lines denote the computed streamlines in a 4:1 contraction.

When the coupling is taken into account, the size of the corner vortex increases [see Fig. 13 and in more detail in Fig. 16(b)]. This observation is confirmed experimentally by Lipscomb et al. [2], who showed an enhancement of the vortex size when slender fibers are added to a Newtonian fluid, even at low Reynolds numbers, and by Yasuda et al. [49], who employed polymeric fibers made from cellulose acetate propionate in a suspension made of glycerin and polyethylene glycol.

The case where the suspending fluid is non-Newtonian and follows a power-law model is now investigated. Figure 14 presents the numerical results for the uncoupled solution. It can be pointed out that the pseudoplastic behavior of the unfilled matrix (characterized with a power-law index of $m=0.3$ ) reduces the upstream vortex, as compared to the Newtonian case. The vortex is pushed completely into the corner and occupies a very little room there, as shown in Fig. 16(c). This outcome has already been confirmed numerically for highly shear-thinning fluid ( $m \leq 0.37)$ [50,51]. As for the fiber orientation field, fibers are mostly aligned along the streamlines.

The results for the coupled solution of fibers suspended in a power-law fluid is given in Fig. 15. The magnitude of the recirculating zone increases slightly with the coupling [see Fig. 16(d)], but its size remains small when compared to the Newtonian cases. It can be concluded that the pseudoplastic behavior of the suspending matrix considerably reduces the

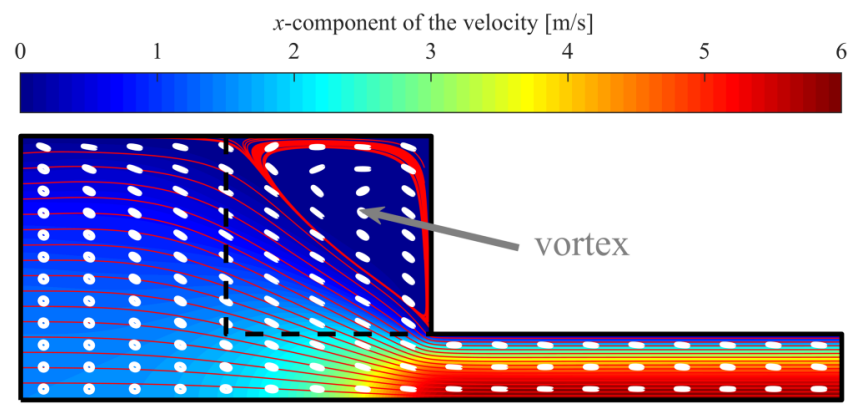

FIG. 13. $x$-component velocity distribution of fluid domain in the $x y$-plane for a Newtonian fluid (coupled solution). Ellipsoids represent the average fiber orientation, and eigenvalues are scaled with $1 / 10$ for better visualization. Red lines denote the computed streamlines in a $4: 1$ contraction.

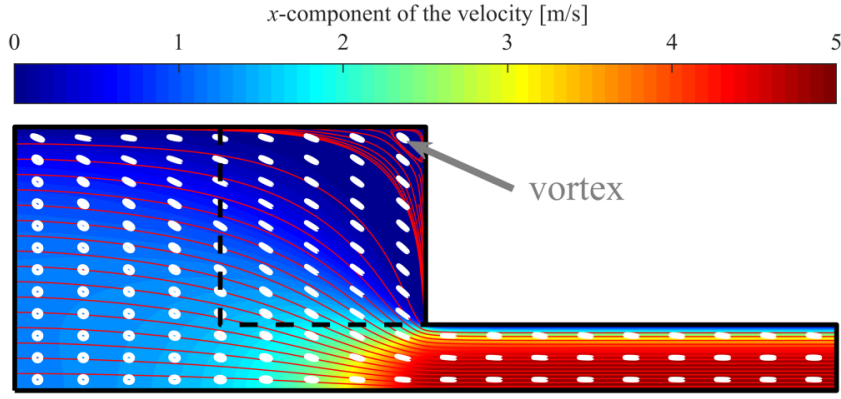

FIG. 14. $x$-component velocity distribution of fluid domain in the $x y$-plane for a power-law fluid with a power-law index of $m=0.3$ (uncoupled solution). Ellipsoids represent the average fiber orientation, and eigenvalues are scaled with $1 / 10$ for better visualization. Red lines denote the computed streamlines in a $4: 1$ contraction.

size of the corner vortex. As observed previously, fibers tends to orient along the streamlines.

In terms of pressure drop, the difference between the uncoupled and coupled solutions is also significant. Figure 17 shows the computed pressure drop along the symmetrical axis (i.e., $y / H=0$ ) in the $4: 1$ planar contraction. By taking into account the coupling effect, the pressure drop increases considerably by at least a factor of 2 independently of the nature of the suspending fluids and under considered simulation conditions. The maximum enhancement is observed for the system involving a Newtonian suspending fluid due to the largest size of the recirculation [see Fig. 16(b)].

\section{TOWARD A SUSPENDING CARREAU FLUID}

It is well known that most melt polymers follow a Newtonian law at low effective deformation rates and then exhibit a pseudoplastic behavior above the attainment of critical effective deformation rates. The shear-thinning and strain-thinning behaviors have been observed for fiber composites made with such suspending matrices [52,53]. In view of this, a simple modeling representation for these materials can be obtained from a bi-viscosity model wherein the transition between viscosities is determined by a critical effective deformation rate condition, $\dot{\gamma}_{c}$. It is then assumed that a composite material follows the Newtonian

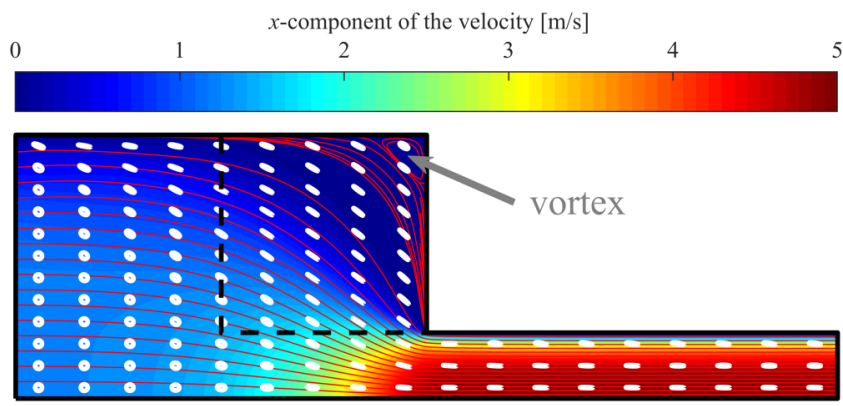

FIG. 15. $x$-component velocity distribution of fluid domain in the $x y$-plane for a power-law fluid with a power-law index of $m=0.3$ (coupled solution). Ellipsoids represent the average fiber orientation, and eigenvalues are scaled with $1 / 10$ for better visualization. Red lines denote the computed streamlines in a $4: 1$ contraction. 
(a)

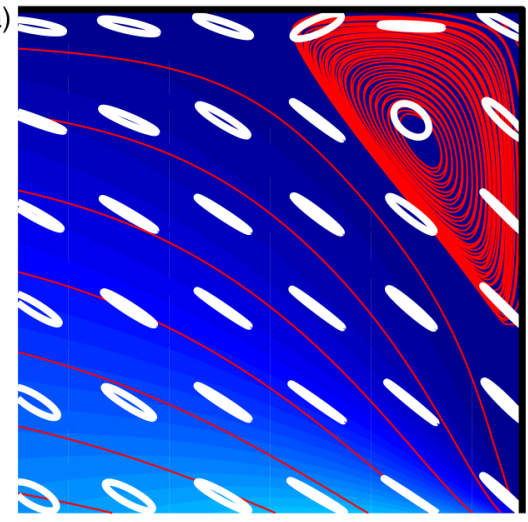

(c)

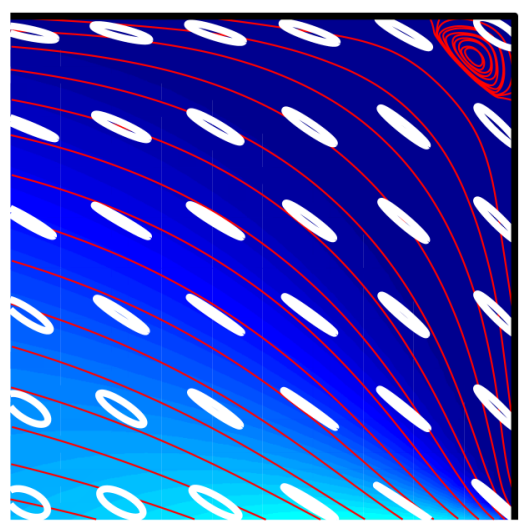

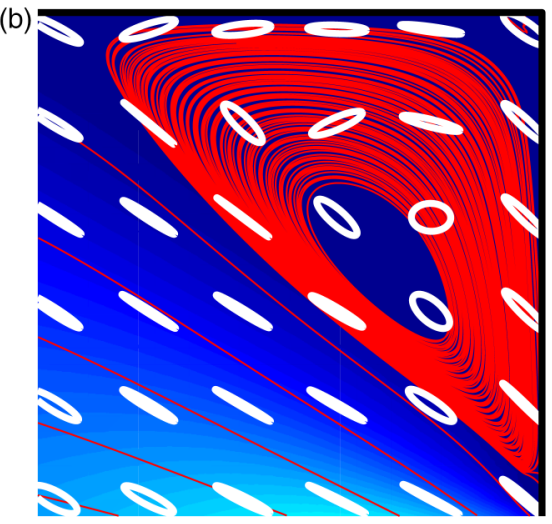

(d)

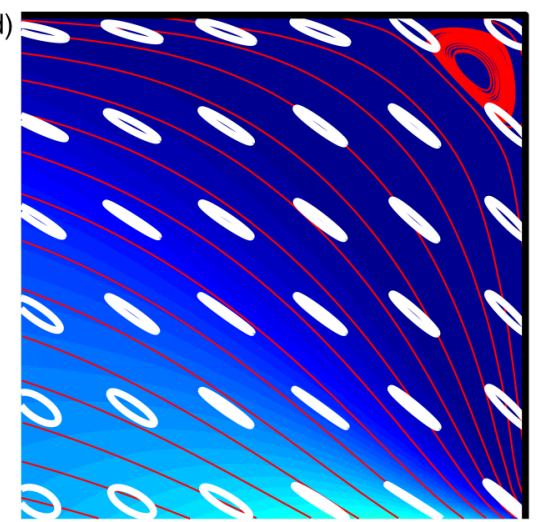

FIG. 16. Higher magnification of the recirculation zones displayed in Figs. 12-15. (a) Uncoupled solution for a Newtonian suspending fluid, (b) coupled solution for a Newtonian suspending fluid, (c) uncoupled solution for a power-law suspending fluid, and (d) coupled solution for a powerlaw suspending fluid.

behavior predicted by Eq. (4) at low $|\dot{\gamma}|$ and the power-law form given by Eq. (3) at high $|\dot{\gamma}|$. This is mathematically summarized below

$$
\begin{gathered}
\boldsymbol{\sigma} \equiv \boldsymbol{\sigma}^{N} \quad \text { for } \quad|\dot{\gamma}| \leq \dot{\gamma}_{c}, \\
\boldsymbol{\sigma} \equiv \boldsymbol{\sigma}^{P L} \quad \text { for } \quad|\dot{\gamma}|>\dot{\gamma}_{c},
\end{gathered}
$$

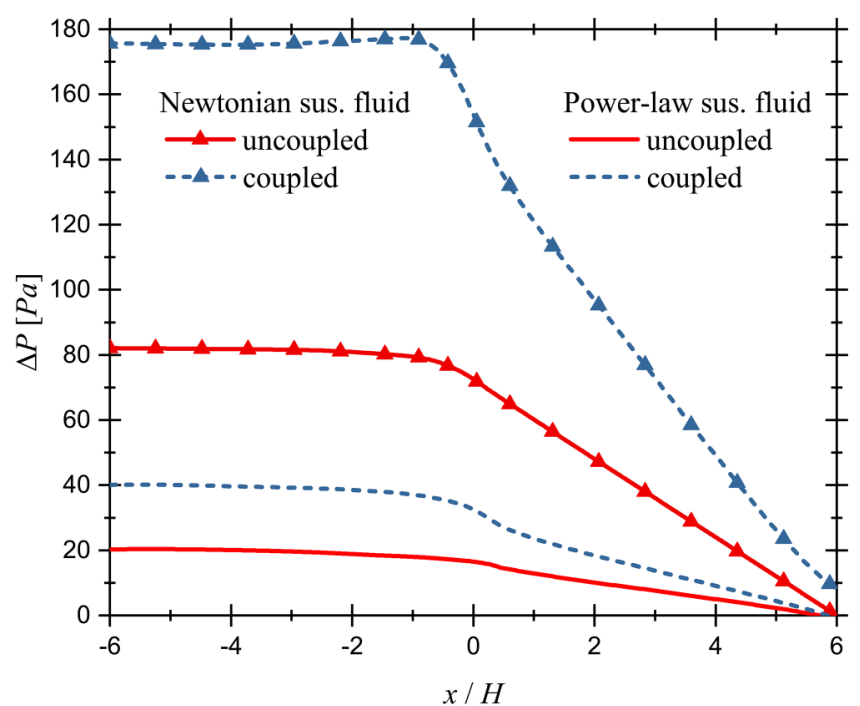

FIG. 17. Computed pressure drop along the symmetrical axis in the $4: 1$ planar contraction. where $\boldsymbol{\sigma}^{N}$ and $\boldsymbol{\sigma}^{P L}$ stands for Eqs. (4) and (3), respectively. In practice, only the power-law model is considered, the Newtonian behavior being obtained by setting the power-law index very close to 1 (i.e., $n=0.99$ ).

The same simulation parameters (as in Sec. IV) have been used and the critical effective deformation rate condition is set to $\dot{\gamma}_{c}=1 \mathrm{~s}^{-1}$. Hence, Fig. 18 (on the top from the black dashed line) shows the regions in the planar contraction, where $|\dot{\gamma}|$ exceeds $\dot{\gamma}_{c}$, or in other words, where the fiber suspension exhibits a pseudoplastic behavior. These regions are located at the contraction entrance and close the wall in the capillary. The associated components of the second-order orientation tensor, $\langle\mathbf{p p}\rangle$, are depicted in Fig. 19 for $\left\langle p_{x} p_{x}\right\rangle$ and in Fig. 20

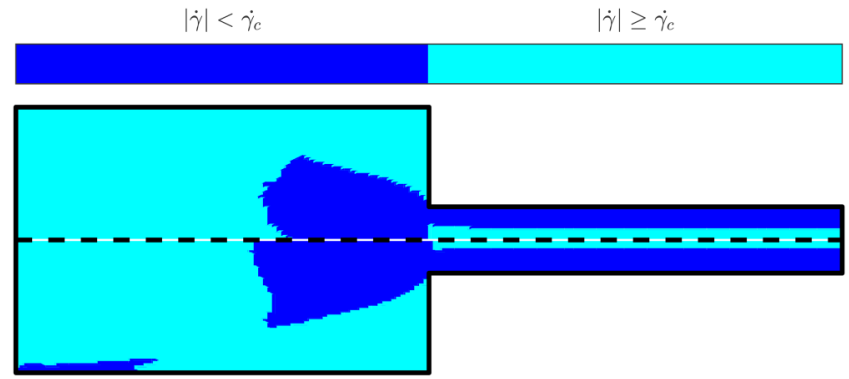

FIG. 18. Predicted regions where $|\dot{\gamma}|$ exceeds $\dot{\gamma}_{c}$ for a coupled problem: the bi-viscosity model on the top and the Carreau model on the bottom. 
0

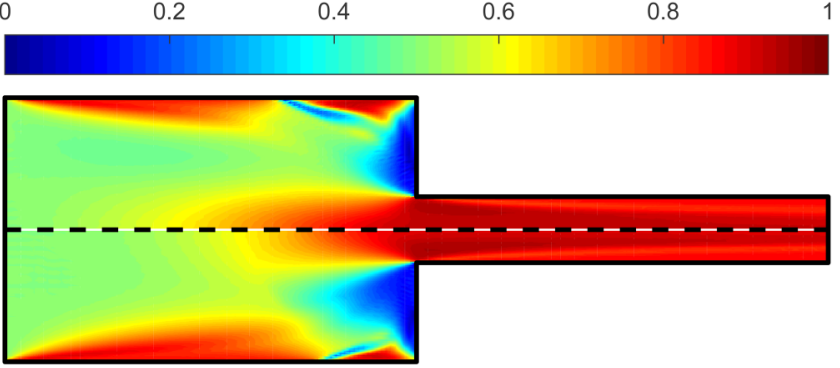

FIG. 19. $\left\langle p_{x} p_{x}\right\rangle$ component pattern representation obtained with a coupled solution: the bi-viscosity model on the top and the Carreau model on the bottom.

for $\left\langle p_{x} p_{y}\right\rangle$ (on the top from the black dashed line), respectively. As previously discussed in Sec. IV $B$, the fibers exhibit a preferred orientation parallel to the streamlines.

In order to deal with pseudoplastic matrices and instead of considering a bi-viscosity model, it is convenient to replace the Newtonian viscosity in Eq. (4) by the one of the Carreau model given by $\eta_{0} \equiv \eta_{0}\left(1+\left(|\dot{\gamma}| / \dot{\gamma}_{c}\right)^{2}\right)^{(m-1) / 2}$. This concept of empirical substitution is useful in commercial injection molding simulation software. With this mind, Fig. 18 (on the bottom from the black dashed line) presents this time the regions, where $|\dot{\gamma}|$ exceeds $\dot{\gamma}_{c}$. As compared to Fig. 18 (on the top from the black dashed line), there are no significant differences between the two patterns, except at the geometry entrance close to the wall. This is explained by a flow rearrangement as the velocity profile at the laminar inflow condition is the one given by the power-law model. For this simulation condition, Figs. 19 and 20 (on the bottom from the black dashed line) show $\left\langle p_{x} p_{x}\right\rangle$ and $\left\langle p_{x} p_{y}\right\rangle$, respectively. Thus, no meaningful differences in term of fiber orientation are observed in connection with Figs. 19 and 20 (on the top from the black dashed line), for which a bi-viscosity model is assumed, except in the size of the recirculating region. From these considerations, it maybe stated that replacing the Newtonian viscosity by the one of the Carreau law in order to take into account the pseudoplastic behavior of the suspending matrix is appropriate if no rapid fiber orientation change along streamlines are encountered (this generally corresponds to a significant geometrical changes in the model).

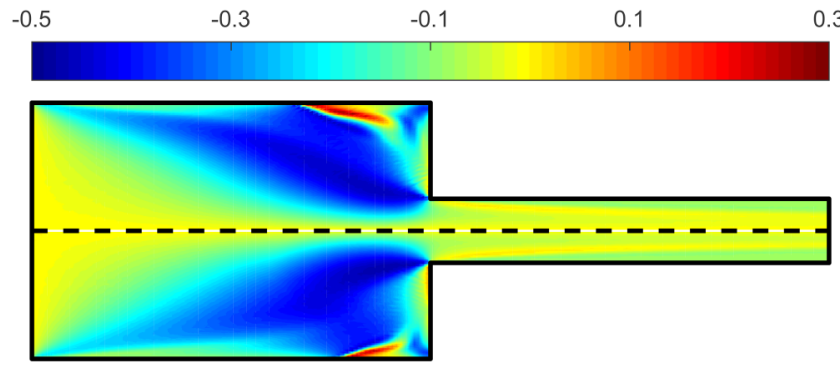

FIG. 20. $\left\langle p_{x} p_{y}\right\rangle$ component pattern representation obtained with a coupled solution: the bi-viscosity model on the top and the Carreau model on the bottom.

\section{CONCLUDING REMARKS}

A numerical method for computing the flow of fiber suspensions with large aspect ratio in planar and converging geometries is proposed. The key point of this work is that the PDF is used to describe the fiber conformation instead of its usual moments. Furthermore, a Newtonian and a nonlinear viscous suspending fluids and their associated particle stress contributions are investigated. The coupling analysis of flow field and fiber orientation shows a close agreement between the coupled and decoupled solutions in terms of fiber distribution. However, the pressure drop increases noticeably with the coupling, being most prominent with the Newtonian suspending fluid. A flattening of the velocity profile is also observed with the coupling and this effect is more pronounced for the Newtonian suspending fluid. The magnitude of the vortex appearing in the corner region for the 4:1 contraction geometry broadens with the coupling. This increase is more noticeable for the Newtonian suspending fluid as compared to the pseudoplastic one. These numerical results are in qualitative agreement with experimental observations available in the literature. Lastly, the Newtonian viscosity is replaced by the one given by the Carreau model. It is found that this empirical viscosity substitution is relevant if no rapid fiber orientation change along streamlines are encountered. This numerical approach will allow us to explore the flow predictions for viscoelastic fluids and fibers suspended in viscoelastic fluids [54], without being hampered by errors arising due to the use of closure approximations.

\section{ACKNOWLEDGMENTS}

D.M. particularly wishes to acknowledge the Brittany Region and the Morbihan subdivision for their financial support.

\section{APPENDIX: EXPRESSIONS FOR $a_{E}, a_{W}$ AND $A_{P}$}

For the upwinding power-law scheme [44], the coefficients $a_{P}, a_{E}$, and $a_{W}$ are determined by

$$
\begin{gathered}
a_{P}=a_{E}+a_{W}+\frac{F_{e}}{2}-\frac{F_{w}}{2}, \\
a_{E}=D_{e} \max \left[0,\left(1-0.1\left|\frac{F_{e}}{D_{e}}\right|\right)^{5}\right]+\max \left[-F_{e}, 0\right], \\
a_{W}=D_{w} \max \left[0,\left(1-0.1\left|\frac{F_{w}}{D_{w}}\right|\right)^{5}\right]+\max \left[F_{w}, 0\right],
\end{gathered}
$$

for which the fluxes are evaluated as

$$
F_{e}=\dot{\phi}_{e}
$$




$$
\begin{gathered}
F_{w}=\dot{\phi}_{w}, \\
D_{e}=D_{w}=\frac{C_{I}|\dot{\gamma}|}{\Delta \phi} .
\end{gathered}
$$

In the above relationships, $\dot{\phi}_{e}$ and $\dot{\phi}_{w}$ represent the rotary motion of a rigid fiber (with $\lambda=1$ ) given by Eq. (1) and expressed at the edges of the control volume.

\section{REFERENCES}

[1] Evans, J. G. V., The flow of a suspension of force-free rigid rods in a Newtonian fluid, Ph.D. thesis, University of Cambridge, Cambridge, 1975.

[2] Lipscomb, G. G., M. M. Denn, D. U. Hur, and D. V. Boger, "The flow of fiber suspensions in complex geometries," J. Nonnewton. Fluid Mech. 26(3), 297-325 (1988).

[3] Ranganathan, S., and S. G. Advani, "A simultaneous solution for flow and fiber orientation in axisymmetric diverging radial flow," J. Nonnewton. Fluid Mech. 47, 107-136 (1993).

[4] Folgar, F., and C. L. Tucker, "Orientation behavior of fibers in concentrated suspensions,” J. Reinf. Plast. Compos. 3(2), 98-119 (1984).

[5] Kabanemi, K. K., J. F. Hétu, and A. Garcia-Rejon, "Numerical simulation of the flow and fiber orientation in reinforced thermoplastic injection molded products," Int. Polym. Process. 12(2), 182-191 (1997).

[6] Reddy, B., and G. Mitchell, "Finite element analysis of fibre suspension flows," Comput. Methods Appl. Mech. Eng. 190(18), 2349-2367 (2001).

[7] Verweyst, B. E., and C. L. Tucker, "Fiber suspensions in complex geometries: Flow/orientation coupling," Can. J. Chem. Eng. 80(6), 1093-1106 (2002).

[8] Yasuda, K., N. Ohara, and M. Muguruma, "Velocity profiles of suspension flows through an abrupt contraction measured by magnetic resonance imaging," Chem. Eng. Technol. 30(8), 1036-1044 (2007).

[9] Mazahir, S. M., G. M. Vélez-García, P. Wapperom, and D. Baird, "Evolution of fibre orientation in radial direction in a center-gated disk: Experiments and simulation," Compos. A Appl. Sci. Manuf. 51, 108-117 (2013).

[10] Mazahir, S. M., G. M. Vélez-García, P. Wapperom, and D. Baird, "Fiber orientation in the frontal region of a center-gated disk: Experiments and simulation," J. Nonnewton. Fluid Mech. 216, 31-44 (2015).

[11] Wang, J., J. F. O'Gara, and C. L. Tucker, "An objective model for slow orientation kinetics in concentrated fiber suspensions: Theory and rheological evidence," J. Rheol. 52, 1179-1200 (2008).

[12] Park, J. M., and S. J. Park, "Modeling and simulation of fiber orientation in injection molding of polymer composites," Math. Prob. Eng. 2011, 105637 (2011).

[13] Advani, S. G., and C. L. Tucker, "The use of tensors to describe and predict fiber orientation in short fiber composites," J. Rheol. 31(8), 751-784 (1987).

[14] Lozinski, A., and C. Chauvière, "A fast solver for Fokker-Planck equation applied to viscoelastic flows calculations: 2D FENE model," J. Comput. Phys. 189(2), 607-625 (2003).

[15] Chauvière, C., and A. Lozinski, "Simulation of complex viscoelastic flows using the Fokker-Planck equation: 3D FENE model," J. Nonnewton. Fluid Mech. 122(1), 201-214 (2004).

[16] Moosaie, A., and M. Manhart, "A direct numerical simulation method for flow of Brownian fiber suspensions in complex geometries," J. Dispers. Sci. Technol. 34(3), 427-440 (2013).
[17] Krochak, P. J., J. A. Olson, and D. M. Martinez, "Fiber suspension flow in a tapered channel: The effect of flow/fiber coupling," Int. J. Multiphase Flow 35(7), 676-688 (2009).

[18] Ammar, A., B. Mokdad, F. Chinesta, and R. Keunings, "A new family of solvers for some classes of multidimensional partial differential equations encountered in kinetic theory modeling of complex fluids," J. Nonnewton. Fluid Mech. 139(3), 153-176 (2006).

[19] Ammar, A., B. Mokdad, F. Chinesta, and R. Keunings, "A new family of solvers for some classes of multidimensional partial differential equations encountered in kinetic theory modelling of complex fluids: Part II: Transient simulation using space-time separated representations," J. Nonnewton. Fluid Mech. 144(2), 98-121 (2007).

[20] Jeffery, G. B., "The motion of ellipsoidal particles immersed in a viscous fluid," Proc. R. Soc. Lond. 102, 161-179 (1922).

[21] Bird, R. B., R. C. Armstrong, and O. Hassager, Dynamics of Polymeric Liquids. Volume 1, Fluid Mechanics, 2nd ed. (Wiley, New York, 1987).

[22] Bay, R. S., Fiber orientation in injection-molded composites: A comparison of theory and experiment, Ph.D. dissertation, University of Illinois at Urbana-Champaign, Champaign, 1991.

[23] Phan-Thien, N., X. J. Fan, R. I. Tanner, and R. Zheng, "Folgar-Tucker constant for a fibre suspension in a Newtonian fluid," J. Nonnewton. Fluid Mech. 103(2-3), 251-260 (2002).

[24] Phelps, J. H., and C. L. Tucker, "An anisotropic rotary diffusion model for fiber orientation in short- and long-fiber thermoplastics," J. Nonnewton. Fluid Mech. 156(3), 165-176 (2009).

[25] Tseng, H.-C., R.-Y. Chang, and C.-H. Hsu, "An objective tensor to predict anisotropic fiber orientation in concentrated suspensions," J. Rheol. 60(2), 215-224 (2016).

[26] Tseng, H.-C., R.-Y. Chang, and C.-H. Hsu, "The use of principal spatial tensor to predict anisotropic fiber orientation in concentrated fiber suspensions," J. Rheol. 62(1), 313-320 (2018).

[27] Laurencin, T., L. Orgéas, P. J. J. Dumont, S. Rolland du Roscoat, P. Laure, S. Le Corre, L. Silva, R. Mokso, and M. Terrien, “3D realtime and in situ characterisation of fibre kinematics in dilute non-Newtonian fibre suspensions during confined and lubricated compression flow," Compos. Sci. Technol. 134(1), 258-266 (2016).

[28] Giesekus, H., "Elasto-viskose Flüssigkeiten, für die in stationären Schichtströmungen sämtliche Normalspannungskomponenten verschieden groß sind," Rheol. Acta 2(1), 50-62 (1962).

[29] Férec, J., E. Bertevas, B.-C. Khoo, G. Ausias, and N. Phan-Thien, "The effect of shear-thinning behaviour on rod orientation in filled fluids," J. Fluid Mech. 798(1), 350-370 (2016).

[30] Férec, J., G. Ausias, and G. Natale, "Numerical evaluation of a single ellipsoid motion in Newtonian and power-law fluids," AIP Conf. Proc. 1960(1), 020006 (2018).

[31] Domurath, J., M. Saphiannikova, J. Férec, G. Ausias, and G. Heinrich, "A model for the stress tensor in dilute suspensions of rigid spheroids in a generalized newtonian fluid," J. Nonnewton. Fluid Mech. 264, 73-84 (2019).

[32] Cintra, J. S., and C. L. Tucker, "Orthotropic closure approximations for flow-induced fiber orientation," J. Rheol. 39(6), 1095-1122 (1995).

[33] Dupret, F., and V. Verleye, Modeling the flow of fiber suspensions in narrow gaps, in Advances in the Flow and Rheology of Non-Newtonian Fluids, Rheology Series 8th ed., edited by D. A. Siginer, D. De Kee, and R. P. Chhabra (Elsevier, Amsterdam, 1999), Vol. 2, pp. 19-29.

[34] Chung, D. H., and T. H. Kwon, "Improved model of orthotropic closure approximation for flow induced fiber orientation," Polym. Compos. 22(5), 636-649 (2001).

[35] Batchelor, G. K., "The stress system in a suspension of force-free particles,” J. Fluid Mech. 41(3), 545-570 (1970). 
[36] Batchelor, G. K., "The stress generated in a non-dilute suspension of elongated particles by pure straining motion," J. Fluid Mech. 46(4), 813-829 (1971).

[37] Goddard, J. D., "The stress field of slender particles oriented by a non-Newtonian extensional flow," J. Fluid Mech. 78(1), 177-206 (1976).

[38] Goddard, J. D., "Tensile stress contribution of flow-oriented slender particles in non-Newtonian fluids," J. Nonnewton. Fluid Mech. 1(1), 1-17 (1976).

[39] Souloumiac, B., and M. Vincent, "Steady shear viscosity of short fibre suspensions in thermoplastics," Rheol. Acta 37(3), 289-298 (1998).

[40] Gibson, A. G., and S. Toll, "Mechanics of the squeeze flow of planar fibre suspensions," J. Nonnewton. Fluid Mech. 82, 1-24 (1999).

[41] Dinh, S. M., and R. C. Armstrong, "A rheological equation of state for semiconcentrated fiber suspensions," J. Rheol. 28(3), 207-227 (1984).

[42] Doi, M., and S. F. Edwards, The Theory of Polymer Dynamics (Oxford Science, New York, 1986).

[43] Férec, J., M. Heniche, M.-C. Heuzey, G. Ausias, and P. J. Carreau, "Numerical solution of the Fokker-Planck equation for fiber suspensions: Application to the Folgar-Tucker-Lipscomb model," J. Nonnewton. Fluid Mech. 155(1-2), 20-29 (2008).

[44] Versteeg, H. K., and W. Malalasekera, An Introduction to Computational Fluid Dynamics: The Finite Volume Method (Pearson Education, Harlow England, 2007).

[45] Chung, D. H., and T. H. Kwon, "Invariant-based optimal fitting closure approximation for the numerical prediction of flow-induced fiber orientation," J. Rheol. 46(1), 169-194 (2002).
[46] Onat, E. T., and F. A. Leckie, "Representation of mechanical behavior in the presence of changing internal structure," J. Appl. Mech. 55(1), 1-10 (1988).

[47] Chiba, K., and K. Nakamura, "Numerical solution of fiber suspension flow through a complex channel," J. Nonnewton. Fluid Mech. 78(2), 167-185 (1998).

[48] Verweyst, B. E., C. L. Tucker, P. H. Foss, and J. F. O’Gara, "Fiber orientation in 3-D injection molded features," Int. Polym. Process. 14(4), 409-420 (1999).

[49] Yasuda, K., S. Henmi, and N. Mori, "Effects of abrupt expansion geometries on flow-induced fiber orientation and concentration distributions in slit channel flows of fiber suspensions," Polym. Compos. 26(5), 660-670 (2005).

[50] Kim-E, M. E., R. A. Brown, and R. C. Armstrong, "The roles of inertia and shear-thinning in flow of an inelastic liquid through an axisymmetric sudden contraction," J. Nonnewton. Fluid Mech. 13(3), 341-363 (1983).

[51] Jay, P., A. Magnin, and J. M. Piau, "Viscoplastic fluid flow through a sudden axisymmetric expansion," AIChE J. 47(10), 2155-2166 (2001).

[52] Chan, Y., J. L. White, and Y. Oyanagi, "A fundamental study of the rheological properties of glass-fiber-reinforced polyethylene and polystyrene melts," J. Rheol. 22(5), 507-524 (1978).

[53] Férec, J., M.-C. Heuzey, J. Pérez-González, L. Vargas, G. Ausias, and P. J. Carreau, "Investigation of the rheological properties of short glass fiber-filled polypropylene in extensional flow," Rheol. Acta 48(1), 59-72 (2009).

[54] Férec, J., E. Bertevas, B.-C. Khoo, G. Ausias, and N. Phan-Thien, "Steady-shear rheological properties for suspensions of axisymmetric particles in second-order fluids," J. Nonnewton. Fluid Mech. 239, 62-72 (2017). 